\title{
Major Facilities for Materials Research
}

\author{
Dean E. Eastman
}

\section{The following is a transcript of Dr. Eastman's Plenary Lecture Presented at the 1984 MRS Fall Meeting}

I am pleased to be here to talk about the work of the Major Materials Facility Committee. A year ago, the President's Office of Science and Technology Policy, in the person of Dr. George Keyworth, who was your 1983 Plenary speaker, asked the National Academy of Sciences to assist the OSTP in establishing priorities for major facilities for materials research. These facilities were loosely defined as costing at least $\$ 5$ million. It was obvious from the beginning that they included sources of synchrotron radiation and steady-state and pulsed neutron sources because neutrons and protons are very prominent probes of matter.

A committee was convened to address this task. Dr. Fred Seitz and I co-chaired the resulting Major Materials Facility Committee, a roster for which is shown in Table I. I'm sure you'll recognize many of the participants as colleagues in physics. chemistry, medicine, and materials science. We began in January 1984, and finished our work in July.* The National Academy of Sciences was very instrumental in helping us to do the job this quickly.

To accomplish the task, we formed four panels to deal with segments of the whole. Because synchrotron radiation facilities and neutron facilities would be of interest, we convened panels to deal with these subjects. We entrusted a third group, under the leadership of Al Narath, to address what we called "other"; that is, to define other facilities to be considered by the group. A fourth group was formed to address budgets, timetables, and associated financial matters.

Before beginning, we had to decide on criteria for priority assessment. The criteria that we developed are listed in Table II.

The first and foremost criterion was importance for frontier research in materials and consideration of needs in biology, chemistry, physics, earth sciences, and medical sciences. Seen here is the interdisciplinary nature of large facilities research. Not only is materials research interdisciplinary, but large facilities for materials research also serve other communities. This, therefore, is reflected in our first criterion. The importance of major facilities for applied research in areas of national priority, economic competitiveness, and security was assessed. Finally, the availability of less costly alternatives and compatability with a long-range national plan for major materials facilities were considered. We developed criteria for evaluating proposals for major facilities from various institutions. (See Table III.)

We also suggested that the construction of major facilities be accompanied by expanded support of smaller materials research programs, including related instrumentation. This requirement, of course, arises because materials research on a medium and small scale provides much of the fundamental new science in the field and trains a large fraction of the manpower involved. This requirement speaks to the question of balance that must be addressed when dealing with such facilities.

The Committee also required that adequate provision for interim support of existing facilities be guaranteed before new facilities proposals be funded. At present a number of large materials research facilities exists, and these are the only user facilities that the United States will have for the next 3 to 10 years. Consequently, we must adequately provide for their operation in the interim. This is a gradual, evolving process.

We also divided new facilities into two categories. The first of these we entitled "Major New Facilities," in which a new facility having little to do with existing infrastructure and its location is contemplated. In this category, we generated a prioritized list of recommendations, as shown in Table IV.

First priority was a warded to the construction of a new $6 \mathrm{GeV}$ synchrotron radiation facility, predicated on optimizing its design for the use of so-called insertion devices; that is, special magnetic units which can be used to increase the brightness orders of magnitude over existing facilities. Second priority is the construction of an advanced steady-state neutron facility, the objective being creation of a flux intensity ten times larger than exists today. The prototype in this category is at the Institut Laue Langevin (ILL) in Grenoble, France, which is one of the newer, and best instrumented, facilities for neutron scattering in the world.

\section{Table I \\ Major Materials Facilities Committee}

Dean E. Eastman, IBM (Co-Chairman)

Frederick Seitz, Rockefeller University (Co-Chairman)

Richard B. Bernstein, University of California, Los Angeles

Robert J. Birgeneau, Massachusetts Institute of Technology

Jerome B. Cohen, Northwestern University

Mildred S. Dresselhaus, Massachusetts Institute of Technology

Harry G. Drickamer, University of Illinois

Peter Eisenberger, Exxon Research and Engineering Company

Donald Engelman, Yale University

Walter Kohn, Institute of Theoretical Physics, Santa Barbara

David W. Lynch, lowa State University and Ames Laboratory

Albert Narath, AT\&T Bell Laboratories

William D. Nix, Stanford University

Edward Rubenstein, Stanford University Medical Center

John J. Rush, National Bureau of Standards

Albert I. Schindler, Naval Research Laboratory

Arthur Sleight, E. I. du Pont de Nemours \& Company, Inc.

William P. Slichter, AT\&T Bell Laboratories

Joseph V. Smith, University of Chicago

Richard Stein, University of Massachusetts

H. Guyford Stever, Universities Research Association

John M. White, University of Texas at Austin

\section{Table II \\ Criteria for Establishing Priorities}

- Importance for frontier research in materials, and consideration of needs in biology, chemistry, physics, earth science, and medical science.

- Importance for applied research in areas of national priority, i.e., economic competitiveness and security.

- Availability of other less costly alternatives.

- Contribution of each facility to a long-term national plan for major materials facilities.

Table III

Criteria for Proposal Evaluation

- Technical and scientific resources at the laboratory.

- Access and ease of use.

- Role of facility relative to mission of laboratory.

- Potential for training scientists and engineers.

Table IV

Recommended Priorities for Major New Facilities

1. $6 \mathrm{GeV}$ synchrotron radiation facility - optimal use of insertion devices.

2. Advanced steady-state neutron facility $-10 x$ flux of existing machines.

3. 1-2 GeV synchrotron radiation facility - optimal use of insertion devices.

4. High-intensity pulsed neutron facility $-\gtrsim 10^{\prime \prime} \mathrm{n} / \mathrm{cm}^{2}-\mathrm{s}$ peak flux, epithermal neutrons.

*A copy of the Committee's report may be obtained from: Major Materials Facilities Committee, Commission on Physical Sciences, Mathematics and Resources, National Research Council, 2101 Constitution Avenue, N.W., Washington DC 20418. 
The third priority is the construction of the new 1-2 GeV synchrotron radiation facility also designed for optimal use of insertion devices. Whereas in the first recommended facility the energy is selected as to emit maximum flux in the hard $x$-ray range, around $1 \AA$, where the wavelength of the $x$-rays are comparable to that of the lattice spacing of matter so as to probe structural questions, the third priority deals with longer wavelength radiation.

The fourth priority is the construction of a new high-intensity pulsed neutron facility where the objective would be a peak flux of $10^{17} \mathrm{n} / \mathrm{cm}^{2}-\mathrm{s}$ of epithermal neutrons. Such a source has a much harder flux of fast neutrons than a steady-state reactor source, and pulsed neutron sources are, in the long run, the only way to exceed the flux limits of steady-state sources.

The second category of priorities, entitled "Recommended Priorities for New Capabilities at Existing Facilities," is listed in Table V.

The first priority, which was unanimously selected, is to take optimal advantage of the two cold sources that are soon to be commissioned at the National Bureau of Standards and Brookhaven National Laboratory, and to install centers for cold neutron research; that is, to install guide tubes, guide halls, and instrumentation to take advantage of developments that have occurred during the last decade. The second priority is to continue the development of additional insertion devices--undulators and wigglers-- that can be installed on several of the existing synchrotron radiation facilities. This was not scheduled to begin until 1987 because there currently is a plan underway to construct a number of these devices, and this plan is adequate through 1986. The third priority is to construct an experimental hall and instrumentation to take advantage of the pulsed neutron source at the Los Alamos National Laboratory. This would provide a source with a peak flux of $10^{16} \mathrm{n} / \mathrm{cm}^{2}$-s, using an existing or soon-to-be commissioned accelerator source. It is an excellent place to gain experience with pulsed neutron sources in addition to the very excellent facility at the Argonne National Laboratory.

The last priority is to install and develop an enriched puised neutron source target to achieve roughly a two- to four-fold increase in the flux of pulsed neutrons. I will return later to other possible facilities that the Committee considered but did not specifically recommend, but first let me show you some of the estimated costs for building these upgraded or new facilities. Table VI, which is taken from the Committee's report, includes initial

\section{Table V}

\section{Recommended Priorities for New Capabilities at Existing Facilities}

1. Centers for cold neutron research - guide halls and instrumentation.

2. Insertion devices on existing synchrotron radiation facilities - undulators, wigglers.

3. Experimental hall and instrumentation at LANL $->10^{16} \mathrm{n} / \mathrm{cm}^{2}-\mathrm{s}$ using WNR/PSR.

4. Upgrade of National Magnet Laboratory - pulsed magnetic fields, instrumentation.

5. Enriched pulsed neutron source target $-2-3 x$ flux increase.

\section{Table VI}

\section{Requirements for New Funding}

Major new facilities (millions of FY 1985 dollars)

$$
\text { In order of priority: }
$$

1. $6 \mathrm{GeV}$ SR facility

2. Steady-state neutron source facility

3. 1-2 GeV SR facility

4. High-intensity pulsed neutron source facility

$\$ 160$

260
70

330

New capabilities at existing facilities (millions of FY 1985 dollars) In order of priority:

1. Centers for cold neutron research

2. Insertion devices on existing SR facilities

\$25-35 each

3. Experimental hall and instrumentation at LANL

4. National Magnel Laboratory upgrade

5. Enriched pulsed neutron target construction costs and a substantial component of instrumentation, without which you have no facility. A raw radiation source or a raw neutron source is useless without suitable instrumentation.

We also gave extensive consideration to the operating costs of such facilities. These are dollars associated with such facilities that are most interchangeable with funding for medium- and small-scale science. We therefore considered operational costs in great detail. For the $6 \mathrm{GeV}$ synchrotron radiation facility, for example, 20 instrumented end stations for conducting research costing a total of about $\$ 35$ million would be used. The operating cost of such a facility would be about $\$ 20-25$ million a year -rather large money to run a rather large facility.

A steady-state neutron source facility, would cost roughly $\$ 260$ million, including instrumentation for 20 state-of-the-art instruments. Likewise, a 1-2 $\mathrm{GeV}$ synchrotron facility that would serve the soft $\mathrm{x}$-ray and vacuum ultraviolet spectral regions and include a dozen end-station instruments is estimated to cost approximately $\$ 70$ million. A high-intensity pulsed neutron source facility would cost about $\$ 330$ million. A very sophisticated accelerator would be required as would about 15 instruments.

New capabilities at existing facilities, also listed in Table VI in order of priority, are led by centers for cold neutron research at either the National Bureau of Standards or the Brookhaven National Laboratory. These include quite a number of instruments and would cost \$25-35 million each. The operating costs of these facilities are essentially negligible. They entail no additional operating expenses beyond those already incurred by those facilities. For insertion devices on existing synchrotron radiation facilities, it would cost about $\$ 20$ million to design, construct, and install about six state-of-the-art storage rings in the United States. The experimental hall and instrumentation required to put in a pulsed neutron facility at Los Alamos to take advantage of the land facilities would be about \$15 million, including several fully operational instruments. The various upgrades required for the pulsed sources at the National Magnet Laboratory was estimated to be about $\$ 5$ million. Finally, puised neutron targets would cost about $\$ 2-5$ million to design, develop, and install.

Before going into more detail about the budgets and timetables, let's consider examples of interesting and unique science that can be done with such facilities. For example, the National Magnet Laboratory in Cambridge, MA, is the only major high magnetic field facility in the country. Many scientific and technology applications experiments in areas of superconductivity, permanent magnets, and semiconductors are conducted using it. It contains several basic types of magnet facilities; DC hybrid magnets can go up to perhaps 35-40 Tesla, although they're a bit below that right now. For higher fields, pulsed systems are used, and improvements in instrumentation are needed for pulsed field facilities of 70 and 75 Tesla. Likewise, the combination of high magnetic fields and very low temperatures (for example roughly 20 Tesla at $\mathbf{5 0}$ milliKelvin) is very important to probe material systems in terms of low effective temperature, and system development is required for such magnetic systems and low temperature systems. (Table VII.)

\section{Table VII}

\section{What is Neutron Scattering, What's New, Why Important}

- The neutron is a unique probe that interacts weakly with nuclei - direct information on the structure of matter as well as its excitations are obtainable from $E, \vec{k}$ scattering measurements.

- Neutrons produced in two ways:

1. In nuclear reactors as by-product of splitting $U$ atoms.

2. With pulsed sources where proton pulses impinge on $W$ or $U$ "targets."

Table VIII

High Magnetic Fields - NML

- Only major high-magnetic-field facility in United States.

- Many scientific and technology applications: superconductivity, permanent magnets, semiconductors, ...

- DC hybrid magnets limited to 35-45T.

- Nondestructive pulsed systems up to $\sim 100 \mathrm{~T},-45 \mathrm{~T}$ and $75 \mathrm{~T}$ system development and instrumentation required.

- Combination of high fields and low temperatures $-197 / 50 \mathrm{mK}$ system development required. 


\section{ILL Facility, Grenoble}

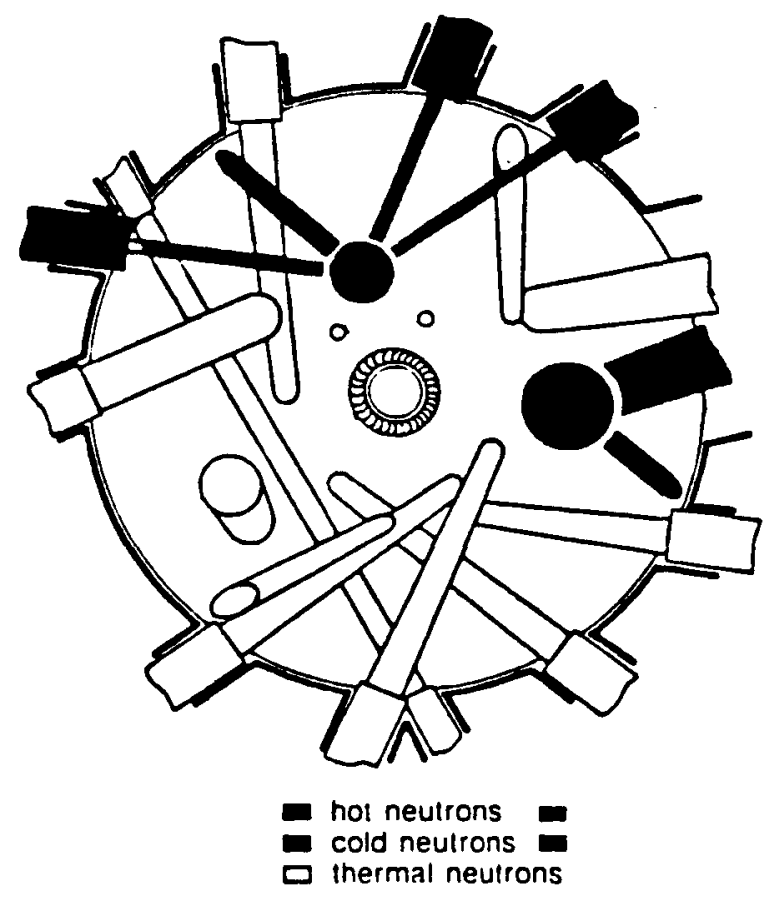

Let me next return to neutron scattering. What is neutron scattering, what's new, and why is it important? The neutron is a unique probe that interacts weakly with nuclei to yield direct information on the structure of matter and elementary excitations from energy-momentum scattering measurements. There are two types of neutron sources. The recommendations deal with both. (See Table VIII.)

In steady-state sources, or nuclear reactors, the neutrons are obtained as a by-product of splitting uranium atoms. In pulsed sources, proton pulses impinge on a tungsten or uranium target, and neutrons are emitted. The rationale for the steady-state neutron source recommendation is that both of the major facilities in the United States today were constructed in the mid-1960s. They're 20 years old now, and if the design were to begin now for a new steady-state facility, it would not be commissioned until perhaps 1998 or the year 2000. By that time the two major facilities in the United States would be some 35 years old. Design and construction of a reactor facility is a lengthy process.

To give you an impression of steady-state sources, Figure 1 is a schematic of the ILL facility in Grenoble. The reactor core is surrounded by a vessel that may be thought of as a reflector of the high density flux of neutrons. Within this vessel are special sources. A cold source, for example, includes another container within the vessel where the neutrons are moderated and slowed to a low energy. The vessel also contains a number of hot or high-energy sources and a variety of guide tubes to extract the neutrons from the sources. Figure 2 illustrates the spectral intensity versus wavelength of various ports or various tubes in the ILL source, and you can see that they extend all the way from a volt or more with a flux of $10^{10} \mathrm{n} / \mathrm{cm}^{2}$ to a tenth of a millivolt far into the long wavelength range. Four to six orders of magnitude in wavelength can be probed using neutrons from such a source.

In general, the energy momentum that can be probed using neutron sources extends from a microvolt up to a volt and wave vectors or momenta from $10^{-4}$ up to $50 \mathrm{~A}^{-1}$, many orders of magnitude. The wavelength is comparable to that of atoms in solids and liquids, and the energy is comparable to thermal excitation energies; very high energy resolution. Neutrons have very different scattering cross sections, for hydrogen and deuterium for example, and various isotopes can be easily viewed, particularly hydrogen.

Neutron scattering is not a static field. If you're not an expert in neutron scattering, you might think that because it's been around for many decades that not much new is happening - quite the contrary. In the last decade,
Mean Flux per Unit Wavelength

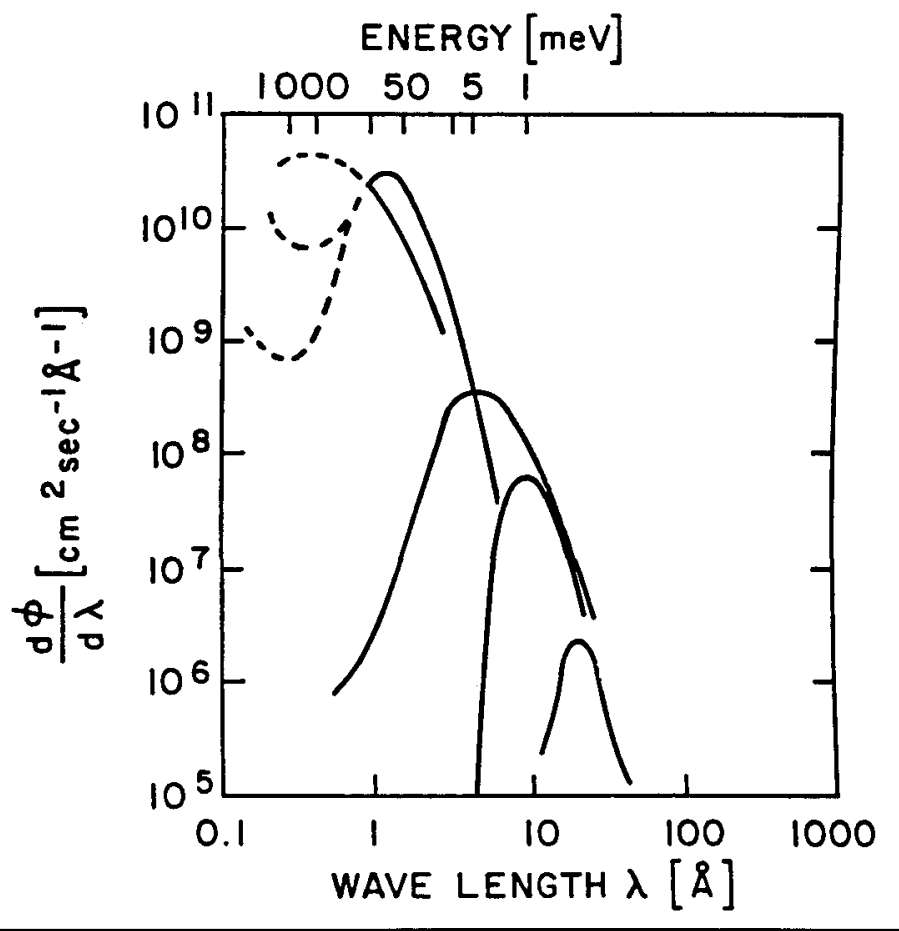

many new achievements have been recorded, as listed in Table IX. Cold sources and neutron guides first appeared about a decade ago. Very high resolution spectrometers, into the microvolt range, were invented some 6-8 years ago. Notably, both of these fundamental accomplishments occurred in Europe, where the user community and the level of activity is substantially larger than in the United States. Many other applications to science, magnetism, powder defraction analysis, interferometry and the like have occurred within the last decade. The advent of cold neutron sources in the higher resolution instruments has spawned great activity within the chemistry community, which has been using these facilities in very large numbers. The small angle neutron scattering instrument at the Oak Ridge National Laboratory has attracted a remarkable number of new users from the polymer community within the last few years.

Figure 3 is a very interesting materials science example of neutron scattering that was presented to our committee, showing measurement of the void volume in a copper sample subjected to fatigue by a periodic stress. The three curves correspond to the three different temperatures. By using small angle neutron scattering, void volumes down to $10^{-6}$ and smaller can be detected nondestructively in copper and various other materials. These

Table IX

Some Major Technical and Scientific Accomplishments of the Last Decade

1974 Cold Sources, Neutron Guides (W. Europe, ILL)

1974 Low Dimensional Magnetism (U.S.A.)

1975 Powder Profile Analysis (Netherlands, ILL)

1975 Neutron Interferometry (U.S.A., Austria)

1975 Charge Density Waves (U.S.A., ILL)

1975 Vibration, Tunnelling \& Diffusion of $H$ in Metals (U.S.A., W. Europe)

1976 Structure of Physi- \& Chemi-sorbed Monolayers (U.S.A.)

1977 High Energy Resolution Spectrometers (W. Germany, ILL)

1978 Bose Condensate in ${ }^{4} \mathrm{He}$; Liq ${ }^{3} \mathrm{He}$ (U.S.A., Canada, ILL)

1979 Coexistence of Magnetism \& Superconductivity (U.S.A.)

1981 Pulsed Spallation Sources (U.S.A., Japan, U.K.) 
techniques are being applied to ceramics and other materials. Table $\mathrm{X}$ lists some of the materials applications of neutron scattering used to probe radiation damage, phase transformations, porosity, polymer structure, and materials processing.

Another example from the area of materials science of polymers is illustrated in Figure 4. Here, small angle neutron scattering from small latex polymer spheres, coated with a $55 \mathrm{~A}$ thickness of a different type of polymer is examined in an experiment to see whether it is possible to resolve the coating and the radial distribution of two dissimilar polymers. What is expected is Bessel function behavior, which is exactly what is seen by the deconvoluted curve in the figure. This is scattering from a very large macromolecule, and is in an area in which neutron scattering is uniquely capable. In fact, the polymer community has been using neutron scattering in recent years in a very substantive way.

Another example is shown in Figure 5. Neutrons are a very unique probe of elementary excitations of matter. In this case the phonon spectra of KCP, a one-dimensional metal, is shown. If you look at frequency, or energy, versus crystal momentum in the Brillouin zone along different crystallographic directions, a kink in the phonon spectra at half the zone boundary where there exists a Peierls distortion and instability. Neutron scattering has long been the standard method of probing phonon spectra, which are among the fundamental excitations of solids upon which we base much of our understanding. Likewise, I could have shown you a spectrum for magnons, the elementary magnetic excitations that neutrons are uniquely suited to probe.

Now let's talk about synchrotron radiation. What is it, what's new, and why is it important? First, the interaction of photons with matter is of fundamental importance and is central to many analysis and processing techniques. These major facilities are primarily aimed at the analysis question, rather than synthesis and processing questions. They're equally important, and in many cases they need to be combined.

Second, synchrotron radiation is a major source of electro-magnetic radiation and is unique, both in its intensity and spectral range. The Lawrence-Berkley Lab Group presented a summary to the Committee of various types of experiments that are done using synchrotron radiation in terms of photon energy from $10^{-3}$ volts up to 100 kilovolts. These experiments probe molecular vibrational excitations, electronic excitations, photoeffect, and $\mathrm{L}$ and $\mathrm{K}$ edges, and on into the hard $\mathrm{x}$-ray regime where structural investigations are conducted. There is a plethora of techniques available using synchrotron radiation.

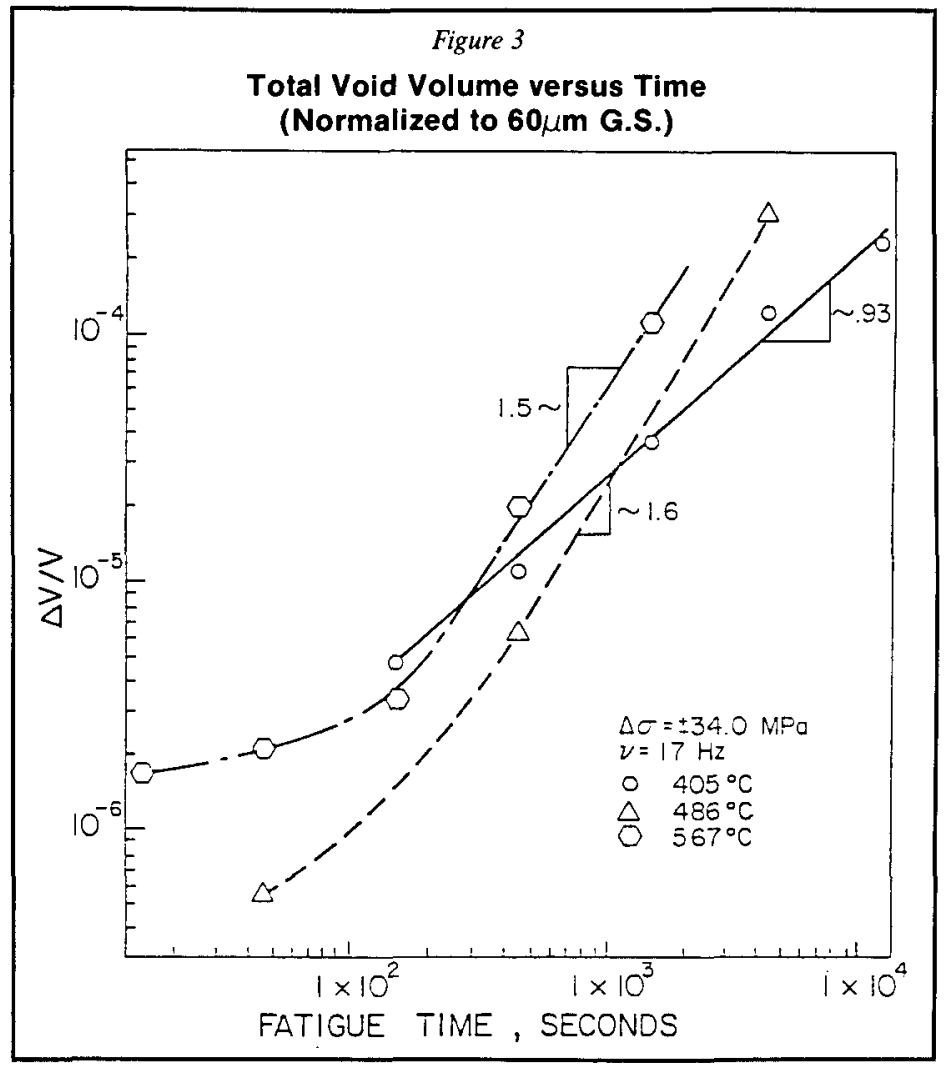

Table $X$

Topics in Materials Science and Engineering Which Have Been Studied by Neutron Scattering

- Damage accumulation:

cavitation

microcracking

microstructural degradation

radiation damage

stress/strain changes

- Residual stress, texture

- Phase transformations

- Porosity (sintering)

- Polymers, colloids, micelles

- Amorphous metals, short-range order

- Materials processing

Figure 4

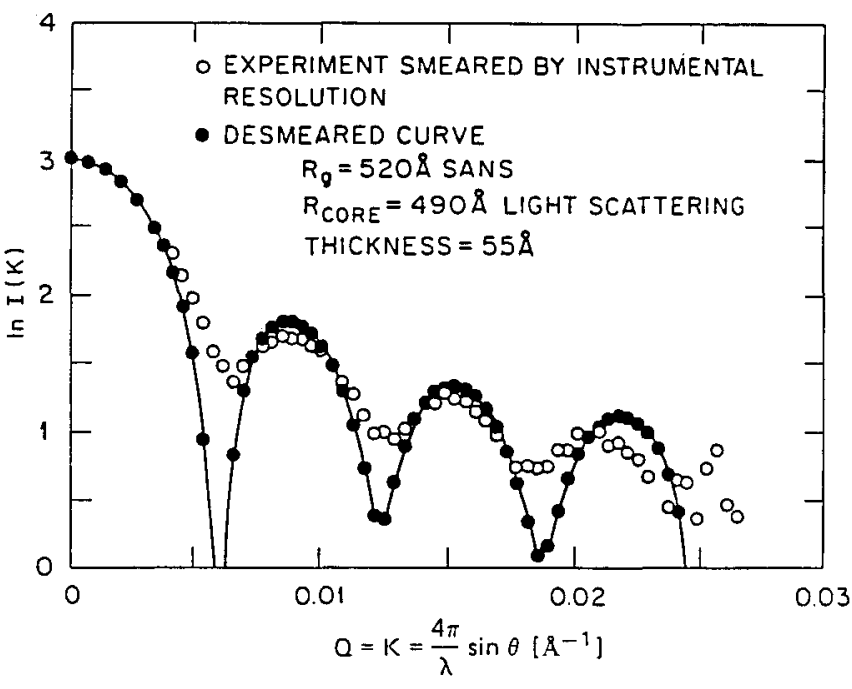

Figure 5

\section{KCP - Quasi One-Dimensional Metal Is Unstable with a Peierls Distortion}

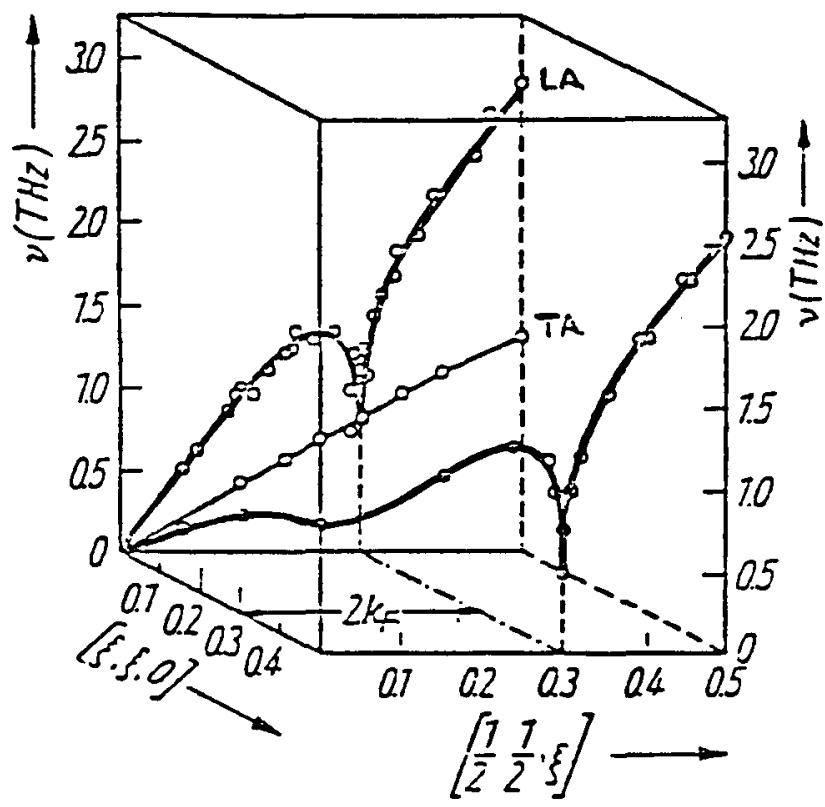


Let me show you what's possible with the recommended facilities, the 6 $\mathrm{GeV}$ facility and a 1-2 GeV facility. By properly designing new sources, extraordinary brightness can be achieved. The electron beam, which is the source of radiation, can be very tightly focused in space to create various kinds of radiation sources. The electrons going around in a synchrotron storage ring source at one of the bending magnets follow a curve so that an arc of radiation shaped like a fan, very small in height but very wide, results. The height is roughly one part in 2,000 to 10,000 . The radiation from a bending magnet is shown in the lower diagram of Figure 6. A hypothetical $1.3 \mathrm{GeV}$ source, which would be an example of priority item 3 , would yield a flux of $10^{14}$ or $10^{15}$ from such a bending magnet. The flux can be increased by a wiggler; i.e., a series of dipole magnets that can bend the beam back and forth, so that the radiation adds up in the forward direction to yield a flux enhancement of one or two orders of magnitude. A few such wigglers are now being started up in the United States. These are much better sources of radiation than bending magnet sources.

Finally, the newest and most sophisticated source is called an undulator. An undulator, like a wiggler, depends upon beam bending, either in a linear polarized mode or in a circular polarized mode, with a series of magnets. In this case, however, the radiation emerges in a quasicoherent way. The laser-like beam can be wavelength tuned with the period of the magnetic structure. As the figure illustrates, the undulator offers a two-decade increase in brightness, or the number of photons per second per square millimeter of source area into the solid angle of emission per band width. Undulator brightnesses approach $10^{19} / \mathrm{s}$ and they're tuneable. These are the sources of the future that we're recommending for major new facilities.

Figure 7 emphasizes how the intensity of $x$-ray sources has increased with time. The reference is an $x$-ray tube. Storage rings represent a many decade improvement, and the addition of wigglers adds another factor of 10 . A newly designed major facility with an optimally designed undulator source nets another 3 or 4 orders of magnitude, an unprecedented intensity.

There are, of course, unknowns. How these various sources interact with one another seems to be sorted out, but has yet to be demonstrated. Also, the flux is so intense that the first optical element can melt if it is not carefully designed. There remain a number of such issues to be considered carefully in design of these sources.

To illustrate their power, consider a 1-2 GeV soft x-ray source. In comparison with the flux obtained from a bending magnet at one of today's facilities, a new source would represent a 100 to 3,000-fold improvement. The significance of this improvement is exemplified by considering an ESCA experiment. Today's conventional $x$-ray tube can deliver $10^{10}$ photons with an $0.2 \mathrm{eV}$ energy spread, which is distributed over a $2 \times 4 \mathrm{~mm}$ sample area. With an undulator source, the sample area can be reduced by a factor of 10 . The energy resolution doubled, and the data collection time reduced 1,000fold. Time-resolved experiments on very small samples are suggested.

This being the Materials Research Society, let me describe an experiment that Ben Larson did several years ago. Ben, of the Oak Ridge National Laboratory, addressed the question of what happens when silicon is laser annealed and melted. What is the process? What is the mechanism? In a coincident experiment, he used a pulsed laser to do the melting, and by timing the pulse with the storage ring source, he did an x-ray scattering experiment, he used a pulsed laser to do the melting, and by timing the pulse with the storage ring source, he did an $x$-ray scattering experiment as a function of delay with roughly 10 nanosecond resolution. He basically looked at the rocking curve of one of the Bragg peaks and was able to map out in time the temperature profile within the silicon as shown in Figure 8 . Twenty nanoseconds after melting, he could determine that for $4,000 \AA$ below the surface, the sample was molten. Fifty-five nanoseconds after the laser pulse, the meltfront had moved halfway toward the surface. Some 155 nanoseconds later, the whole sample had recrystallized and temperature profile greatly flattened. This is a very elegant example of the use of synchrotron radiation. After speaking about an experiment in the nanosecond range, l'll just mention that in one of the presentations to the Committee, it was proposed that the recommended sources will be able to resolve events of order of 10 picoseconds instead of 10 nanoseconds. Thus, the time resolution of such experiments will be greatly extended.

It is very difficult to pick out examples of this sort because for every one included, I leave out scores of others. This last example, therefore, is also representative of many more. It is called an $x$-ray microprobe, by analogy with the electron microprobe. There is a large number of electron microprobes in the United States today-somewhere between 1,200 and
2,500 . Over a billion dollars has been spent on electron microprobes in this country. Electron probes are one of our primary characterization tools, but rather than using electrons, $x$-rays and soft $x$-rays from the recommended sources can be used to do the same type of work. A 1-2 GeV ring would make a soft $x$-ray microprobe instrument, while a $6 \mathrm{GeV}$ ring would produce a hard $x$-ray source. The properties of this latter instrument are compared with the capabilities of an advanced microprobe. (See Table XI.) The minimum detectable mass is improved by about a factor of 5,000 for a $I \mu \mathrm{m}$ probe size. The minimum probe size for the $x$-ray case would be around $500 \AA$. For smaller probe sizes of course, electrons, which can be focused much better than $\mathrm{x}$-rays, are preferred. The number of electrons or $\mathrm{x}$-rays needed for the same detectability heavily favors $x$-rays, whose cross sections are much larger than those of electrons. This is the basis for a very interesting analytic probe of matter, using $\mathrm{x}$-ray or soft $\mathrm{x}$-ray sources. Levels of detectability would be extended to $0.01 \mathrm{ppm}$, and would mean it would be possible to go in and look at the electrically active doping elements in semiconductors, which cannot be done today in the electron microprobe. Today we use SIMS, a destructive technique; the $\mathrm{x}$-ray probe would allow nondestructive examination of semiconductor doping, grain boundary diffusion, and a host of surface treatment related experiments.

Having looked at a few examples of what can be done with these sources, let us now consider the connection between materials research sub-areas and other fields that utilize major facilities. Materials research, I believe, is a very

\section{Figure 6}

\section{Bending Magnet/Wiggler/Undulator Comparison}
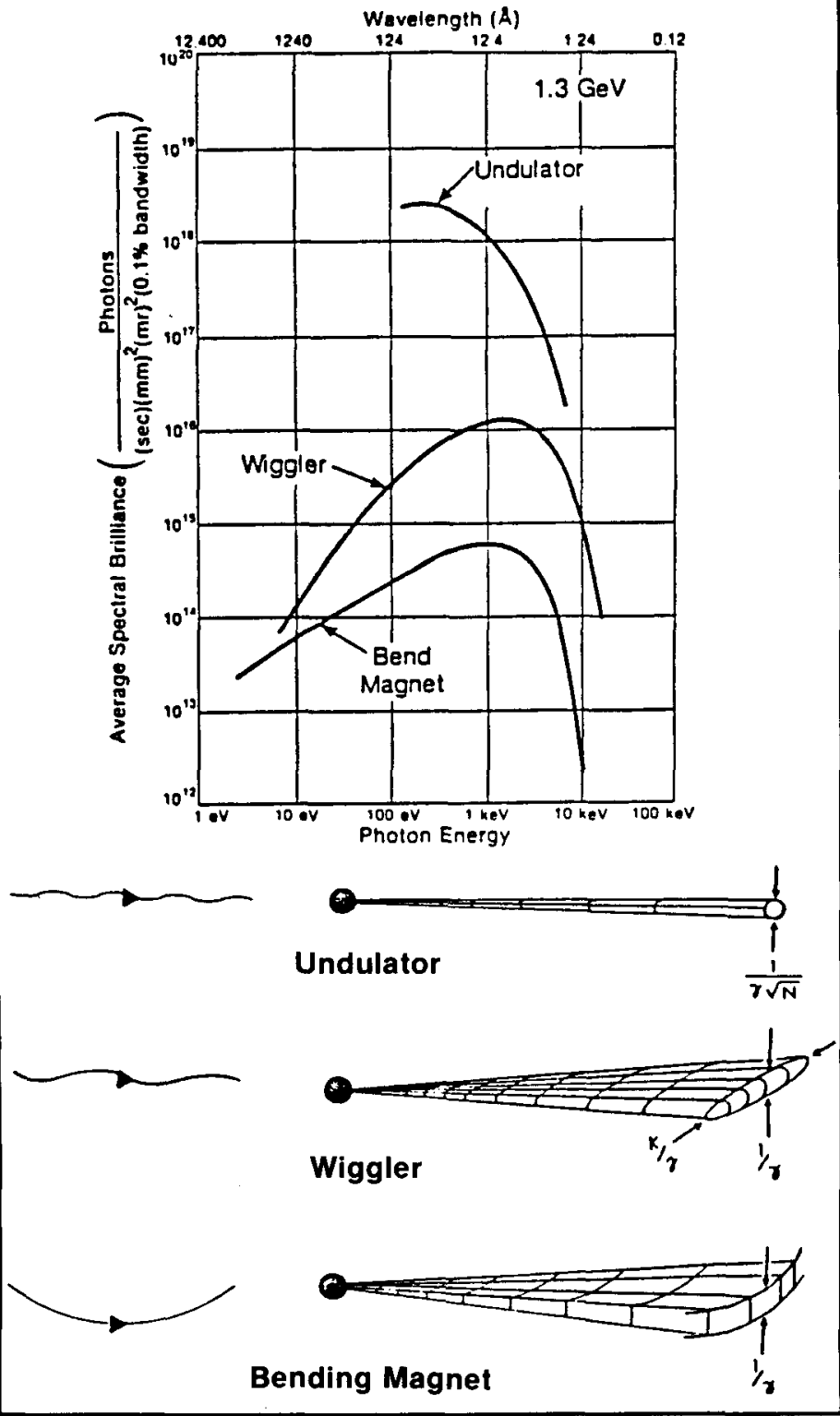
Figure 7

\section{History of X-ray Sources}

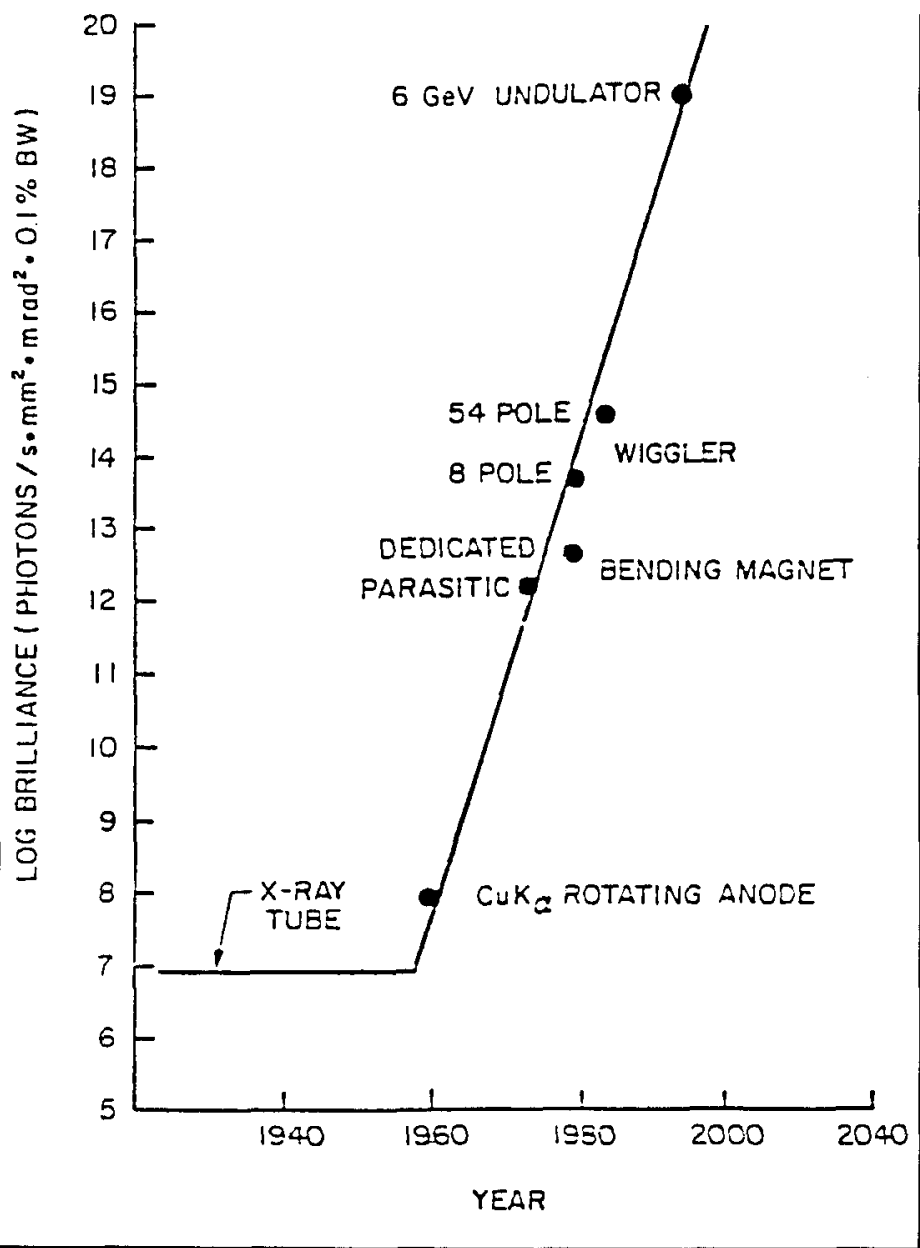

unique area of research in that it has both technological significance and importance in terms of fundamental science. It's one of the very intellectually active and fertile areas of science. You can judge that very simply by the number of Nobel Prizes won in materials research, and the number of new concepts and new activities that come out of materials research. It provides underpinnings of much of our advanced technology. It is one of the few areas, I believe, where industry is a principal participant in conducting both applied research and also basic research.

Similarly, materials research impacts many areas of national interest, as Table XII illustrates. This chart, taken from the Committee's report, shows the impact of various areas of materials research on information systems, energy, health, national security, and transportation. The marks indicate that these areas of materials research are important to those technological and defense-related areas, an impressive testimony to the value of materials research.

Let us now consider the issues of time and money. Figure 9 also taken from the Committee's report, shows the funding profile for construction of three major new facilities; a $6 \mathrm{GeV}$ ring, an advanced nuclear reactor, and a 1-2 $\mathrm{GeV}$ ring. Expenditure is shown to begin at the earliest possible date, assuming that the design phase is begun immediately. These profiles are not the Committee's recommendations, but rather an estimate of the time and money that would be required. The $6 \mathrm{GeV}$ ring, for example, would require 5-6 years to build, while the advanced reactor construction would require a decade. Finally, the 1-2 GeV ring would be completed more quickly.

In the case of new capabilities at existing facilities, less money and typically three years are required, as Figure 10 indicates. For example, in the case of synchrotron radiation undulators, development is already approved through 1986. We estimated that six new sources could be built over a period of about three years. The tallest bars represent the total construction profile for all three of the priorities together. Again, the Committee did not recommend that all of these be funded together, or necessarily in this time sequence.
Figure 8

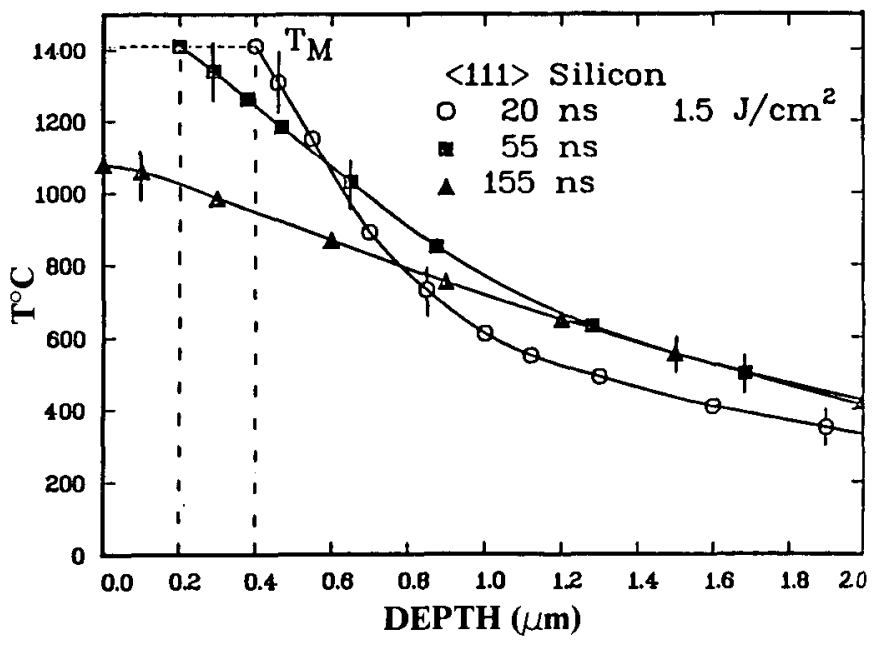

What does this all mean? Our recommendations comprise hundreds of millions of dollars, but must be compared to historical trends. The Committee took a look at U.S. investment in major facilities over the last 20-25 years, beginning in the 1960 s when the neutron facilities that are used today were constructed. If we measure everything in 1985 dollars to put it on a common footing, the U.S. investment, primarily in neutron facilities, over the last 25 years has been roughly $\$ 800-850$ million, or about $\$ 40$ million per year of construction funds over the last 20-25 years. By comparison, the Committee's list represents an expenditure in construction of about $\$ 50$ million a year. If one takes into account that the subject of synchrotron radiation is an area that didn't exist 20 years ago, and that the national audience of users is probably 6-10 times larger than it was two decades ago, the total funding profile of the recommendations is not out of line with maintenance of current U.S. investment in major facilities.

We also discussed and debated whether or not some of these expensive facilities might not be better shared, for example, with our European friends, rather than being constructed in the United States. The Committee felt that in the case of a state-of the-art $6 \mathrm{GeV}$ facility, a state-of-the-art steady-state reactor that would really provide capability for the latter part of the century, one could really not afford to forego having at least one such instrument in the United States. I should point out that one of the facilities not on the funding graph is the pulsed neutron facility, which is the most expensive. It was omitted because not enough is known about the use of pulsed neutron sources, which are relatively new. Our recommendations were that development continue at Argonne National Laboratory and be started at Los Alamos National Laboratory so that we understand and better appreciate applications for pulsed neutron sources. We recommended that any pulsed neutron facility be considered at a later date.

What else did we consider? I gave you the original charge to the Committee regarding the definition of major facilities. It is not unique. We, therefore, convened a panel to address other kinds of facilities that might be considered and be given priority consideration. Considered, debated, and discussed at length among the Committee members and subpanels were facilities of the type listed in Table XIII. First, we considered medium-scale, specialized facilities, for example, materials research laboratory type organizations or special purpose materials research laboratories, such as ones dealing with polymers, ceramic research, and processing. In fact, the Committee heard a number of presentations dealing with such hypothetical facilities, and excellent arguments were put forward for a number of them. Also considered were large electron microscope facilities, ion beam facilities, and microelectronics fabrication facilities such as those in North Carolina, Cornell, and elsewhere.

We also looked at other specialized photon sources; free electron lasers, and vacuum UV lasers, which are new developments. We also looked at some length at computational facilities, which are also very important for materials research. We considered muon facilities, and we discussed the whole issue of instrumentation, which is very important for materials research as well as other fields. Medium-scale, specialized facilities, despite excellent arguments that were heard for them, were excluded from the report 
without judgment, mainly because or their open-ended nature. I nat is, the Committee felt that these facilities are collections of various smaller instruments and concepts. They are open-ended in nature, and as such, require fuller definition through specific proposals by specific institutions, and a central committee cannot decree what type, flavor, kind, or number of such open-ended facilities could be effective. We, therefore, excluded them without judgment, although it was the consensus of many of the Committee members that many of these warrant equal attention with the subset of facilities that we did specifically consider.

Some of the other items listed were either smaller in size and dollars, or premature. Computational facilities are being dealt with currently by both the National Science Foundation and the Department of Energy, and the Committee applauded those undertakings. Likewise, there is a section in the report dealing with the question of instrumentation, which is very important. The major materials facilities that I've been describing may be regarded as specialized types of instruments, or really collections of instruments, useful for many. They supply the country with a capability, but are by no means the whole of materials research. Medium-scale instruments, for example, in the $\$ 50,000$ to $\$ 3$ or $\$ 4$ million range, such as molecular beam epitaxy systems, ion scattering systems, and the like, are equally important. These are not within the charter of the Committee.

In closing, I quote from the introduction to the Committee's report: "A continuing concern throughout our Committee's deliberations was and still is the possible misinterpretation or misuse of the report. As we emphasize throughout the report, the knowledge generated by materials research comes from diverse research styles and participants. Each is vital to the total effort and complementary to each other. These various styles all must be well supported for the health of the total effort in materials research. Consequently, one cannot-because this question of balance is very important-view major materials facilities as more important than other sectors. One should view it as a complement, as an important part of the whole. Therefore, the recommendations in this report should be read and interpreted for what they are: statements as to priorities solely for major materials facilities. We did not address any judgments on the total spectrum of work embraced by materials research. By the same token, our Committee recommends in its report that additional panels be convened to address other aspects of materials research and to review previous recommendations in light of new information. Such panels, by responding to specific aspects of materials research needs, can contribute to the long-range planning and the setting of priorities for the materials research field."

We did not recommend, although some of our members felt that we should, that a master committee be appointed to recommend priorities for all of materials research. The majority of the Committee did not feel that materials research as a whole can be dealt with by a committee such as HEPAC, in the high-energy physics community, where there's a relatively small number of decisions to be made. Materials research is too diverse, and for that reason we specifically limited the charter of our activity.
Figure 9

Estimated Costs: New Facilities

Fiscal Years 1985-1996

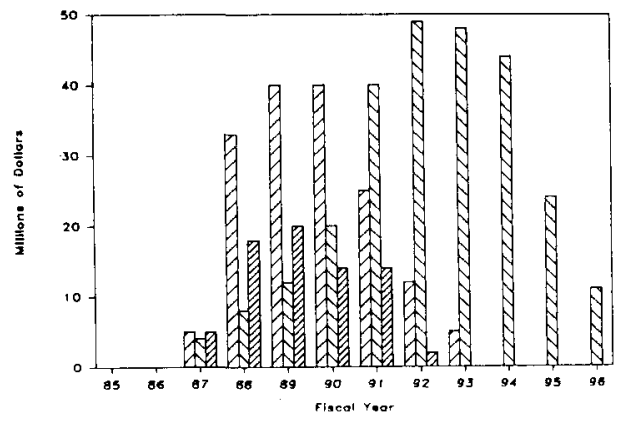

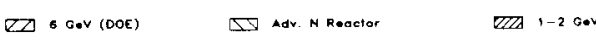

Figure 10

Estimated Costs: New Capabilities Fiscal Years 1985-1996

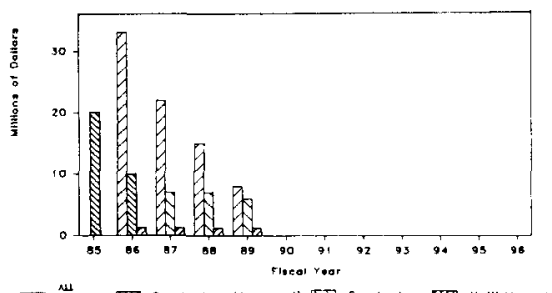

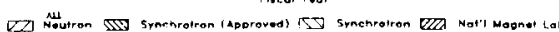

Table XIII

Other Specialized Facilities

Considered by the Committee

Table XI

\section{Comparison of a Proposed X-ray Microprobe with Most Advanced Electron Microprobes (6 GeV Ring)}

Characteristics

X-rays

Electrons

Minimum detectable mass fraction $\mathrm{s}^{-1}$

for $1 \mu \mathrm{m}$-diam probe

Minimum detectable mass

$\mathrm{s}^{-1}$ for 500.1 probe

Minimum spatial resolution (samples

$100 . X$ to $2000 . X$ thick)

Number of electrons and $x$-rays

for the same MDMF

Relative signal to background (contrast)

Relative thick-target fluorescent yields

\section{$0.01 \mathrm{ppm}$}

$50 \mathrm{ppm}$

\section{0 atoms}

10,000 atoms

$5001 \quad 10$ to 500 i

1

$10^{3}$

$10^{4}$

10 to 150
1

1
Table XII

Connection Between Materials Research Sub-Areas and Other Fields that Utilize Major Materials Facilities and Technological Applications of National Interest

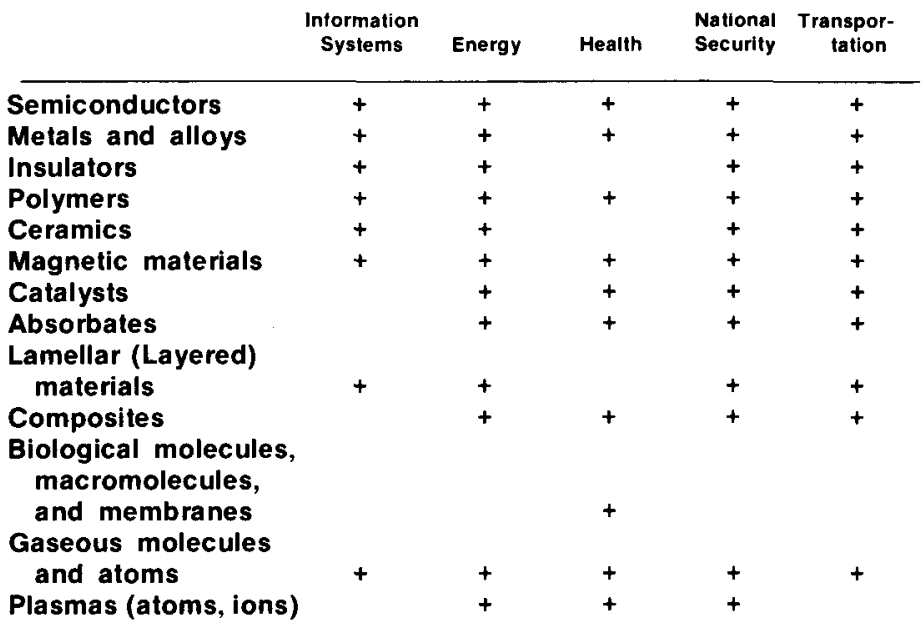


Now on Sale

\section{FALL MEETING PROGRAM AND ABSTRACT BOOK}

This compact reference which contains an index to authors and chairs is an excellent compendium to the 14 proceedings and extended abstracts volumes to be published from the Meeting.

Send check for $\$ 5.00$ to:

Publications Department

Materials Research Society

9800 McKnight Road, Suite 327

Pittsburgh, PA 15237

telephone: (412) 367-3003

See details on proceedings and extended abstract volumes inside the back cover of this issue.

\section{MRS Members Offered Reduced Subscription Rates to AIP Journals}

As an affiliated Society of the American Institute of Physics, the Materials Research Society is able to offer its members reducedrate individual subscription to AIP journals.
To order subscriptions at these rates, send your subscription order, with remittance and a statement indicating your MRS membership, to:

Subscriptions

American Institute of Physics

335 East 45 th Sreet

New York, NY 10017

\section{AIP JOURNAL SUBSCRIPTIONS}

\begin{tabular}{lrrrr}
\hline \multicolumn{1}{c}{ Journal } & \multicolumn{2}{c}{ Domestic Rates } & \multicolumn{2}{c}{$\begin{array}{c}\text { Foreign Rates } \\
\text { Incl. Canada/Mexico }\end{array}$} \\
\hline & Member & Nonmember & Member & Nonmember \\
\hline Journal of Applied Physics & $\$ 80$ & $\$ 450$ & $\$ 126$ & $\$ 496$ \\
Applied Physics Letters & 40 & 260 & 63 & 283 \\
The Journal of Chemical Physics & 100 & 760 & 160 & 820 \\
Journal of Mathematical Physics & 50 & 440 & 66 & 456 \\
The Physics of Fluids & 45 & 395 & 65 & 415 \\
Physics Today & 20 & 50 & 33 & 63 \\
Review of Scientific Instruments & 30 & 250 & 45 & 265 \\
Current Physics Index & 65 & 290 & 81 & 306 \\
Journal of Physical and Chemical Reference Data & 50 & 200 & 60 & 210 \\
General Physics Advance Abstracts & 12 & 150 & 24 & 162
\end{tabular}

